\title{
Perceived movement of the afterimage during eye movements'
}

The question of whether an afterimage viewed in a dark field appears to move during eye movement was studied by comparing recordings of eye movements with recordings of reports of perceived movement. The correlation was found to be quite good even under conditions where the eye movements were spontaneous rather than specifically directed. The results were taken to support the hypothesis that the behavior of the retinal image is "interpreted" by taking into account information concerning what the eyes are doing.

"When the eyes move, the after-image moves in the same way [Helmholtz, 1924]." Since Helmholtz, if not before, it has generally been assumed that an afterimage would appear to move if the eyes were moving while it was viewed. The general basis of this notion is the phenomenon of position constancy: the fact that despite movements of the $O$ that cause the image to move over the retina, a stationary scene will continue to look stationary. It is plausible to believe that position constancy is the result of a process in which image displacement that is concurrent with movement does not lead to the perceived movement of the objects whose images are displacing, because under these conditions the displacement is taken into account and movement is attributed to the $O$ himself. Thus, image displacements dependent on $O$ movement are taken into account so that the objects in the visual field appear stationary and the $O$ experiences himself or some part of himself to be moving. Now, if it is the case that a correspondence between image displacement and $\mathrm{O}$ movement is a necessary condition for position constancy, then the absence of such a correspondence should lead to the loss of position constancy and the appearance of movement in the visual field. Since the afterimage does not displace, the afterimage should appear to move when the $\mathbf{O}$ moves.

One might summarize this view of position constancy by saying that retinal image displacements are evaluated in terms of the available information about $O$ movements so that a correspondence between image displacement and $O$ movements will always lead to position constancy and conversely a lack of correspondence, retinal displacement in a noncorresponding direction or at a noncorresponding rate, or the absence of any retinal displacement at all coupled with $O$ movement will always lead to the appearance of object movement. The perception of an afterimage during eye movement is quite analogous to Emmert's Law in the case of size perception. If the diminishing size of the image is taken into account in perceiving size as constant at increasing distances, then a nonchanging image must be seen as changing size with increasing distance.

Some qualifications: The evidence now indicates that the information about eye position and eye movement is derived from signals corollary to those going to the eye muscles rather than from proprioceptive feedback from the eye muscles (Brindley \& Merton, 1960). Thus, where there is no efferent information that the eyes have been directed to move and there is image displacement-as in the case of a passively pushed eye viewing a normal scene-no transference of the displacement "energy" occurs-and the scene will appear to jump. An afterimage, however, viewed under the same condition should appear stationary, for there is neither efferent information about eye movement nor image displacement. ${ }^{3}$

It has recently been demonstrated that abnormal image displacement coupled with head movement invariably leads to the perception of movement, at least prior to adaptation to this unusual state of affairs (Posin, 1966; and described in Rock, 1966; Wallach \& Kravitz, 1965). If the image displaces at a different rate or in a different direction than the head, it will appear to move. Only if it displaces at the same rate and in the normal direction will it appear stable. This has not, however, been unequivocally demonstrated with abnormal image displacement accompanying eye movement. Wallach and Lewis (1965) report that a mismatch between image displacement and eye movement most often does not lead to reports of movement. By a technique entailing the perception of the shadow cast by the pupil of the eye, these investigators achieved a state of affairs in which the movement of the shadowed disc was controlled by the movement of the eye, so that it moved only when the eye moved. The technique made it possible to produce a situation in which the target disc moved across the retina at either a faster or slower rate than the rate at which the eye was moving. This experiment led Wallach and Lewis to conclude, "Our results are incompatible with the view that the apparent rest of visual objects whose images shift due to eye movements is to be explained by a compensating process which takes the eye movement into account [p. 28]." Instead they argue that any shifting of the image during movements of the eye is simply disregarded or suppressed. Yarbus (1967), however, reports that abnormal image shifts coupled with eye movements do, in fact, lead to the appearance of object movement.

"If the direction of the retinal image shift in step with the saccade was not directly opposite to the direction of movement of the retina, the visual object also appeared to be moving jerkily [p. 207]."

Yarbus's results, which differ from the Wallach and Lewis findings, appear to be based on the use of specially designed contact lenses that enabled him to vary the direction of image displacement accompanying eye movements.

Thus, while an investigation into the relationship between eye movements and the apparent behavior of the afterimage might have seemed like an investigation of the obvious, there now appears to be reason to question whether in fact the absence of image displacement matched with eye movement necessarily must lead to the perception of movement. If it is true that there generally is no perception of movement when the magnitude or direction of displacement of the retinal image no longer corresponds to the magnitude or direction of movements of the eye, then one might reasonably predict that a condition of nondisplacement of the image accompanying eye movement might also fail to yield the perception of movement. "No displacement" is, after all, a special case of abnormal or nonmatching behavior of the image when coupled with an eye movement. On the other hand, if the taking-into-account process that is effective in maintaining position constancy during head movements also operates during eye movements, we should predict that an afterimage will be seen to move. 
It is, of course, also possible to argue that no displacement of the retinal image accompanying eye movement is a special case and that it is only necessary that there be some unspecified retinal image displacement for position constancy to obtain. On this view the correlations between eye movement and retinal image movement signifying position constancy would be completely nonspecific so that any direction or rate of image displacement during an eye movement should be accounted for by the eye movement. The difficulty with this view is that it fails to account for the ability to perceive movement during an eye movement.

Since a distinction can be made between the perception of a change in visual direction and the perception of movement per se (the hour hand of a clock regularly changes its visual direction without our normally being aware of any movement), and visual direction certainly must be a function of eye position and retinal location, the operation of a taking-into-account process can only be established by reports of afterimage movement, not simply by reports of shifts in the visual direction of the afterimage, as the eyes move.

The experiment to be described is an attempt to determine whether in fact the afterimage does appear to move when the eyes move. The experiment is, to our knowledge, the first actually to investigate this matter. Prior statements in the literature appear to be based only on informal observation, the results of which may sometimes be misleading.

\section{Apparatus}

The experiment had at least two major requirements: an afterimage that was sufficiently long lasting to permit us to investigate its apparent behavior, and a means for simultaneously recording the movements of the eyes and the O's perception of the movement of the image. The afterimage was produced by a $400-W$ bulb at a distance of 18 in. from the O's eyes. A two-channel Beckman Dynograph with a dc amplifier was used to record the data.

Throughout the experiment the $O$ sat with his head within a specially constructed box $14 \mathrm{in}$. high, $18 \mathrm{in}$. deep, and 26 in. wide, the open side of which was fitted with a bite board that the $O$ used during testing. A heavy black cloth attached to the top of the box shrouded the $O$ 's head and upper torso and created a light-tight cell. The experimental room itself was dark except for the light, in most cases a small tensor lamp, that was necessary for E to monitor the recorder.

The experiment had two phases. During the first phase the 0 was given training in tracking a sporadically moving and disappearing target light. He had to keep a long horizontal arm continually pointing at the target. Preliminary experimentation indicated that the reliability of the data could be increased by such training since tracking an afterimage that fades and reappears is not an easy task. The training also had the advantage of allowing Os to become proficient in the use of the apparatus, and it provided us with important information about the lag in responding to a moving target. The second phase of the experiment involved the tracking of the afterimage. In both phases, the $O$ continuously indicated the direction of the target, whether the light appeared to be stationary or not. The equipment used by the $O$ to report the behavior of the light was located on the lower surface of the box. A potentiometer located on the base of the box was wired through a battery to one channel of the recording apparatus. The shaft of the potentiometer carried a foot-long arm, oriented horizontally, that the $O$ was instructed to grasp with his right hand and to move in order to indicate the apparent position of the target light or afterimage. The $\mathrm{O}$ was instructed to have this arm always pointing directly at where he saw the light. To aid the $O$, tacks were placed in the base of the box along the arc in which the pointer moved at the $0-, 45-, 90-, 130$-, and 180-deg marks. The $O$ was able to feel these as he moved the pointer. The zero position of the pointer coincided with the $O$ 's straight ahead. The arm could be moved through an arc of about $200 \mathrm{deg}$ ( $100 \mathrm{deg}$ either side of straight ahead). A telegraph key was placed to the left of the pointer on the base of the box. It was wired to one of the two event markers in the recording system. The 0 was instructed to depress the key whenever the light (target or image) appeared to be stationary.

A second potentiometer was secured to the top of the box directly above the center of the bite board. From the shaft of this potentiometer a metal arm extended $1 \mathrm{ft}$ just beneath and parallel to the upper surface of the box. At the end of this arm was a $11 / 2-V$ light bulb behind a milk-glass plate bearing a 1/2-in.-diam hole. The housing of the bulb was light-proof so that, while the light was on, the $O$ saw only a small disc of diffuse light insufficiently bright to illuminate any features of the box that had been painted matte black to minimize reflectance. At the top of the shaft and outside the box a knob was installed that allowed the $\mathrm{E}$ to move the light. This light, which was very close to the $O$ 's eye level, was visible through an arc of about $200 \mathrm{deg}$. This light was the target light and was used only during the training phase of the experiment. Any movement of this light was displayed on one channel of the recorder. A switch was inserted in the wiring of this channel in order to enable $E$ to couple this channel to either the target potentiometer or to the eye electrodes. Thus, during Phase 1 the two main channels recorded target and pointer movement, while during the second phase lateral eye movements and pointer movement were recorded. The second event marker recorded whether the target light was on or off and was later correlated with the $O$ 's verbal report of whether or not the light was visible. This marker did not function during Phase 2.

The eye electrodes, which were placed at the temples of the $O$, picked up potential differences with a sensitivity ranging from .2 to $1 \mathrm{mV} / \mathrm{cm}$. Paper speed was maintained at $1 \mathrm{~mm} / \mathrm{sec}$ throughout testing with the exception that when the instructions were given during Phase 2 to move the eyes quickly the speed was increased to $5 \mathrm{~mm} / \mathrm{sec}$. This facilitated the reading of the eye movement records. The recording system was calibrated so that the extreme positions of the target light, pointer, and as far as possible the eyes, corresponded with the extremes on the recording paper; the straight-ahead location of the light, pointer, and eyes corresponded with the central position on the recording paper.

The afterimage projector was placed outside the rear wall of the box. On this wall, directly in front of the eyes of the $O$, was a small hole which housed a shutter mechanism $1 \frac{1}{4}$ in in diam. With the shutter closed and the projector light on, enough light leaked through the aperture to enable the $O$ to fixate the shutter. (This light was on only immediately preceding and during the creation of the afterimage.) The afterimage was produced by a $1 / 4-\mathrm{sec}$ exposure to the intense projector light. Only one pulse of light was given at a time and was not repeated until the $O$ reported the total disappearance of the afterimage.

\section{Method}

Conditions of testing differed somewhat for Os. These differences and the reasons for them will be made clear. All Os began the testing procedure with Phase 1, target tracking. After being settled as comfortably as possible with their heads within the testing cell, the $O$ was told that his task was to keep the pointer, which he grasped in his right hand, constantly pointing in the direction in which he saw the target light. He was also told that any time he did not see the light move, he was to press down 
the telegraph key located next to his left hand. The E carefully explained to the $O$ the difference between seeing something move and seeing a change in its position. An analogy was made to the hands of a clock in order to make this distinction as clear as possible. Instructions to the $\mathrm{O}$ emphasized the importance of this distinction and throughout the testing $\mathrm{O}$ was reminded of it. $\mathrm{O}$ was also asked to report "fade" whenever the light was not visible. It was extremely important that the $O$ remember to report fade during Phase 2 whenever the afterimage disappeared, since a failure to do so would have left us with a misleading instance of a negative correlation between eye movement and the perception of image movement. The eye movement record could not possibly be mirrored by the tracking record if the $O$ had no image to track.

When it was clear that the $O$ understood the instructions, Phase 1 testing began. The target was switched on, the recording device was calibrated, and the $E$ began to move the light in a more or less random fashion. Intermittently, and again randomly, $E$ would turn the target off. Any time the $O$ reported fade, it was marked on the recording paper, and these reports were later checked against the record of target on or off. Phase 1 lasted approximately $5 \mathrm{~min}$ and was directly followed by Phase 2 . Phase 2: Os were told that they would have to look at a briefly pulsed intense light that would leave them with an afterimage. They were instructed to report the behavior of the image in the same way that they had reported the behavior of the target light. When the Os reported that the image had completely disappeared, on the average of 2 to $3 \mathrm{~min}$ after exposure, a new afterimage was produced. This process was repeated until the day's testing was completed. Immediately before the afterimage light was pulsed, $O$ was required to fixate the dim light at the rear of the box, directly in front of him. Exposure was binocular (monocular exposure results in a less durable afterimage), and all testing was done with the eyes open in the dark testing cell.

All sequences of afterimage testing began with directions simply to report what was seen with the pointer and telegraph key (nondirective instructions or ND). For Os 1-5 these instructions were followed at intervals by specific directions to move their eyes in a particular way, the sequence of these instructions being varied from $O$ to $O$ and from one testing day to the next for the Os who were tested more than once. These instructions were: "Move your eyes slowly left and right as directed" (slow controlled or SC). "Move your eyes slowly any way you please" (slow uncontrolled or SUNC). "Move your eyes quickly any way you please" (fast uncontrolled or FUNC). "Move your eyes quickly left and right as directed" (FC). "Look straight ahead" (straight ahead or SA). Finally, "Inspect the contours of the afterimage" (inspect or I). It was necessary to instruct all Os to look straight ahead from time to time in order to recalibrate the machine and correct for problems of "drift," a variable characteristic of dc energy that manifests itself on the record as a gradual movement of the recording pen in a particular direction independent of the on-going eye movements.

Observers 6-11 were tested primarily under ND instructions, although for a few of these Os directed eye movements (I, FUNC, and SUNC) were introduced during the final minutes of testing, and they were occasionally given SA instructions. The reason for this change was a suspicion that repeated instructions to move the eyes might be biasing the $O$ 's reports. There was reason to believe that at some points instructions to move the eyes were also, perhaps unconsciously, acting as instructions to move the pointer. Evidence of this was the fact that during specific eye-movement instructions the record of pointer movement was occasionally either prior to or synchronous with the eye movement. Observers $1,2,4,5,9,10$, and 11 were also given passive eye-movement instructions while viewing an afterimage. These Os were asked to

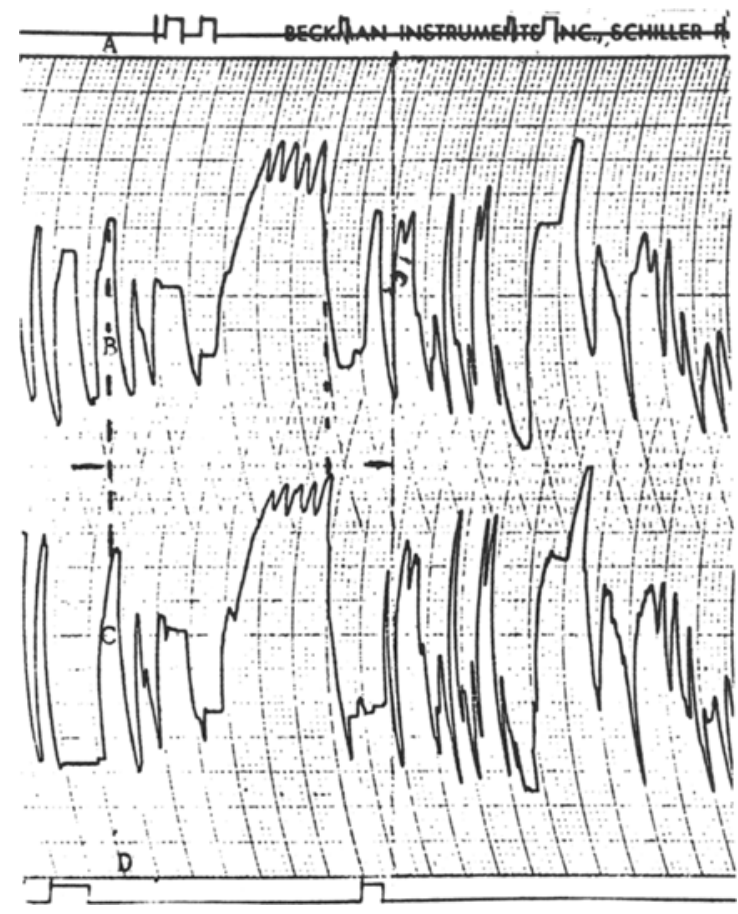

Fig. 1. Line A: Event marker record of whether light was seen moving or stationary. Indentations indicate that no movement was perceived. Line B: Target light. Up indicates movement to the left and down indicates movement to the right. Line $C$ : Pointer movement record. Directions the same as Line B. Line D: Event marker record of target light on and off.

Paper speed $1 \mathrm{~mm} / \mathrm{sec}$. Dotted line indicates a point of correspondence between target light and pointer.

report the behavior of the image while gently pushing either their left or right eye with their finger. These instructions were always last in the afterimage testing sequence.

\section{Observers}

Eleven Os, college students, were tested. They were paid volunteers. Observers 2 and 3 were tested repeatedly for 5 days. Observers 4 and 5 were tested twice on 2 different days. The remainder of the $11 \mathrm{Os}$ were tested only once. Repeated testing of some Os was done in an attempt to increase their precision in reporting, a difficult task at best. This did not prove sufficiently effective to warrant repeated testing of all Os, particularly since there was some chance that repeated testing might, in fact, lead to more automatic and less observant reporting.

\section{Results}

Phase 1: Target tracking reports. The records of target tracking indicate an extremely high correlation between the target's movement and the $O$ 's ability to report that movement with the pointer. The lag in his response to target movement was at most $3 / 4 \mathrm{sec}$ and was usually between $1 / 4$ and $1 / 2 \mathrm{sec}$. Os quite reliably reported the absence of movement with the telegraph key and their reports of target light disappearance matched the record of target light off sequences. In all but a very few instances, when the target light moved in a particular direction to a particular extent, the Os reported movement in that direction and to that extent. The rare exceptions may, we think, be attributed to lapses of attention. Such lapses of attention are hardly surprising even in Os who are trying their best to do what is asked of them, since 


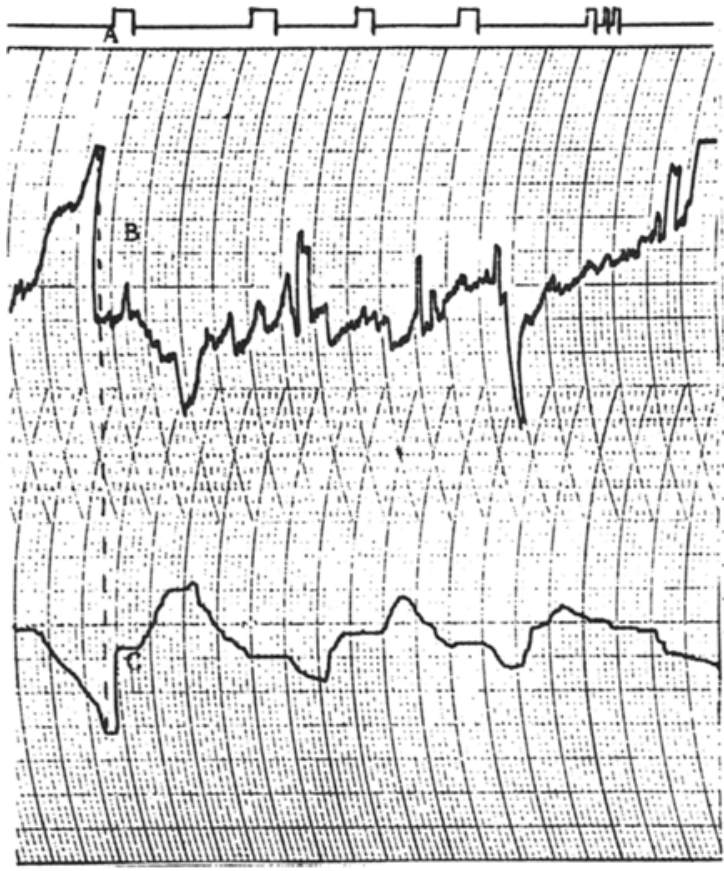

Fig. 2a. Line A: Event marker record of whether afterimage was seen moving or stationary. Line B: Eye-movement record. Up indicates movement to the right; down indicates movement to the left. Line C: Pointer movement record. Up indicates movement to the left; down indicates movement to the right.

Paper speed $1 \mathrm{~mm} / \mathrm{sec}$. Dotted line indicates a point of correspondence between the eyes and the pointer.

the task is both tedious and demanding. This problem is far more severe in the afterimage sequence where the $O$ is asked to monitor the action of a less distinct and at times barely visible target that may be changing its shape and color before his eyes.

These results provide an index against which the afterimage records may be compared, with the proviso that reporting the behavior of an afterimage is a more difficult task. Figure 1 presents a section of a typical target-tracking record illustrating the degree to which target movements were reported. Of particular interest is the extent to which target light disappearance and a change of position without the perception of movement were reported. Target light disappearance can be checked against the record of "fade" reports, while no movement reports are indicated by the event marker column on the extreme left of Fig. 1. The perception of a position change accompanied by no apparent movement should have been reported whenever the target light was momentarily switched off and moved. Thus, the accuracy of these reports can be checked by comparing the record of the telegraph key used to report no apparent movement and the record of target light on or off that appears on the extreme right column of Fig. 1 .

Phase 2: Afterimage tracking. The record of eye movements and image tracking indicates a fairly good, if sometimes gross, correlation. Perhaps the purest evidence of this is seen in the records of image movement reported during the initial period of inspection when $O$ had been given either ND or SA instructions for Os 1 to 5 and the records of Os 6 to 11 in their entirety. During these testing periods there were no recurrent references to movements or specific directions to move the eyes that might have biased the reports. For reasons stated earlier, records of directed eye movements are best considered inconclusive and we have ignored them in evaluating the data.

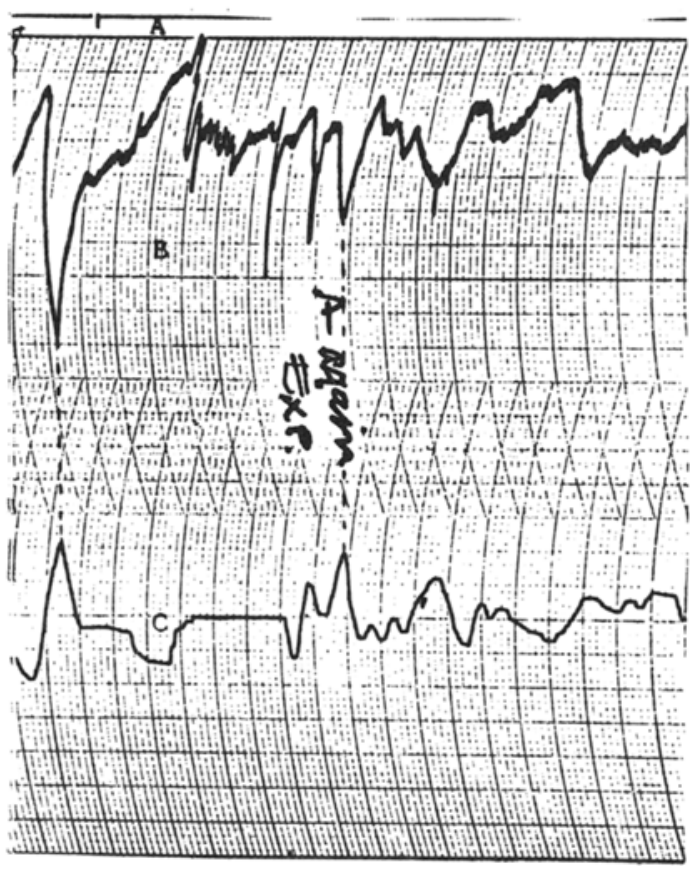

Fig. 2b. Line A: Event marker record of whether afterimage was seen moving or stationary. Line B: Eye-movement record. Up indicates movement to the right; down indicates movement to the left. Line C: Pointer movement record. Up indicates movement to the left; down indicates movement to the right.

Paper speed $1 \mathrm{~mm} / \mathrm{sec}$. Dotted line indicates a point of correspondence between the eyes and the pointer.

If we ignore all instances of afterimage tracking obtained under conditions of instructed eye movements, the remaining records reveal that about $80 \%$ of the time the subjective impression of image movement was mirrored by the record of eye movement. We are, of course, excluding from consideration the many instances where the afterimage faded. Figures $2 a$ and $2 b$ are examples of afterimage tracking. Figure 2a presents an instance of image tracking during $\mathbf{S A}$ instructions. Figure $2 \mathrm{~b}$ is an example of image tracking during ND instructions. It is important to note in examining these figures that left-right directions are reversed in the eye-movement channel, so that a correlated record is one in which the data lines are more or less symmetrical and the mirror image of each other. It is of interest to compare these figures with Fig. 1 and to notice that the lag between eye movement and the report of image movement in Figs. $2 a$ and $2 b$ closely resembles the lag between target movement and the report of movement in Fig. 1. It is, we believe, evident from these records that the apparent movement of the image is strictly dependent on the movement of the eyes.

About $20 \%$ of the time our records reveal either no correlation, a negative correlation, or a poor correlation between image and eye movement. The most frequent instances of no correlation occurred during periods in which the $O$ had been instructed to look straight ahead. Some of these records reveal drifting or small saccadic movements of the eyes that are not mirrored by pointer movement. Since these kinds of eye movements led to reports of image movement elsewhere in our records, the absence of a correlation here needs to be explained if the taking-into-account thesis is to be maintained. At least a partial explanation may be that the primary efferent eye-movement information during these episodes is that the eyes are stationary and straight ahead. If this were the case, a taking-into-account theory would predict no 
apparent image movement, for there would be no available information that the eyes had moved. No explanation can be given of the rare instances of negative correlation between eye and image movement-instances in which the image is reported moving in a direction opposite to the direction of eye movement. These instances are rare, however, accounting for less than $1 \%$ of the negative data.

Instances of poor correlation are those in which the $\mathrm{O}$ reports either somewhat more or less image movement than the recorded eye movement. These instances appear to be more or less comparable to those instances in the target-tracking data in which the records of pointer movement do not reproduce the actual target movement. We have attributed this lack of perfect correlation to lapses of attention on the part of the $O$ and believe that lapses of attention also account for the majority of instances of poor correlation in the afterimage data. While instances of poor correlation occurred less than $5 \%$ of the time during target tracking, they occurred slightly more frequently during afterimage tracking.

The record of perceived image movement does not reflect eye movements in precisely the same way that the record of perceived target movement reflects actual target movement. The record of perceived image movement tends to be a smoothed out rather than an exact replica of eye movement. Frequently saccadic excursions of the eyes that occurred during a gradual shifting in eye position seem to be ignored. If several of these saccades occur close together, then they seem to be summed, resulting in a report of image movement in the direction of the saccades.

The records of perceived image movement obtained during those periods in which the movements of the eyes were specifically instructed by the $E$ consistently show an excellent (sometimes too excellent) correlation between eye and image movement, but for reasons already stated this evidence is given less weight than that obtained during periods of spontaneous eye movement.

Passive eye movement. None of the seven Os who were asked to gently push their eyes right or left report image movement in the same direction as the passive eye movement. Several Os reported a slight movement of the image in the opposite direction, which may have been the result of actively initiated movements back to a resting position. Since it was not possible for Os in this condition to manipulate the pointer while pressing their eye, the reports of image movement were verbal and transcribed directly on the record by the $\mathbf{E}$.

\section{Discussion}

The fact that the apparent movement of the afterimage is dependent on and determined by eye movements directly supports the thesis that position constancy during eye movements is a function of a taking-into-account process in which retinal displacements are nullified if matched by corresponding eye-movement information. These data appear to contradict those recent findings that would argue against such an hypothesis (Wallach \& Lewis, 1965; Stoper, 1967).

One might want to argue that eye movements merely produce a change in visually perceived direction, and it is this change in direction that the $O$ mistakenly reports as movement of the afterimage. Os were, however, trained to distinguish between instances of position change and instances of movement. Proof that they were able to make this distinction may be found in target-tracking data where the record of telegraph key pressing-the O's means of reporting no apparent movementmatches the instances in which the target light was momentarily switched off and moved. Here there was a change of direction without apparent movement. Further proof may be found in the reports of afterimage behavior following instructions to move the eyes rapidly right and left. These instructions resulted in sweeping saccades and frequently led to subjective reports that the image seemed to discontinuously change its position, e.g., "I see it [the image] only at the beginning and the end" (that is, when the eyes came to rest at an extreme right or left position). Saccadic suppression often led to reports of position change without movement, indicating the $O$ 's awareness of this distinction. We believe it is safe to conclude that whenever the 0 reports image movement, he is actually experiencing movement and not merely a position shift. If we are correct to conclude that our findings and those of Wallach and Lewis are not compatible (however, see below), then it would be desirable to reexamine the effect of abnormal shifting of the image during eye movement using techniques other than those used by Wallach and Lewis.

The question of the kind of eye movements producing an impression of movement of the afterimage is a relevant one. Wallach and Lewis argue that the instances where their Os did see the abnormally displacing image moving occurred only during intentional eye movements. It was during incidental eye movements based on instructions to check "the edges of the disc for sharpness on all sides" that their Os did not see movement. That being the case, it is interesting to note that we, too, are relying primarily on the data derived from more incidental and nondeliberate eye movements, namely, those occurring under ND and SA instructions. Stoper (1967) emphasizes the difference between saccadic and pursuit movements, claiming that only during the former does position constancy obtain. Our results show that the afterimage is seen to move during saccadic eye movements. Strictly speaking, we have no instances of pursuit movement, since there is no actually moving object to follow. But we do have many instances of slow or drifting movements of the eye and these, too, are usually accompanied by the experience of afterimage movement.

Stoper (1967) argues that a taking-into-account hypothesis (which he calls the cancellation theory) cannot account for the perception of movement during pursuit movements of the eye because staionary objects in the background do seem to move when the eye follows an object moving in the field (the Filhene illusion). ${ }^{4}$ He presents additional evidence of this fact in the case of stroboscopic movement. He reasons that, if there is no discounting of the retinal displacement of stationary objects during pursuit movements, then the perceived movement of the "pursued" object can hardly be based on a taking-into-account process. He offers instead an account of the perceived movement of the fixated object during pursuit movements based on a "pure motion" signal originating in the oculomotor system (p. 180).

If we ignore the distinction between saccadic and pursuit movement, then the case of a "pursued" object in an otherwise dark field is identical to the case of an afterimage viewed by a moving eye. In both instances there is eye movement and no retinal displacement. Stoper's theory, therefore, may be applicable to our results. It could be argued that the "pure motion" signal theory also predicts the apparent movement of the afterimage so that our results cannot decisively support a taking-into-account hypothesis.

If, as Stoper suggests, we should separate the explanation of how a displacing image is perceived from that of how a stationary image is perceived during one and the same eye movement, then perhaps our results do not contradict those of Wallach and Lewis. They found that an abnormal displacement of the image during eye movement does not lead to the perception of movement. We find that the special case of abnormal image behavior during eye movement, namely no movement at all, is seen as movement. But we prefer the more parsimonious hypothesis that one mechanism, a taking-into-account process, explains how a displacing and a 
stationary image are perceived and, from that point of view, we are puzzled by the difference between our results and the Wallach and Lewis results, and we are puzzled by Stoper's data showing no position constancy during pursuit eye movements. ${ }^{5}$

The apparent movement of the afterimage during eye movements cannot be explained on the basis of movement detectors, i.e., of cells in the visual system, that fire if, and only if, an image moves across the retina. Rather this finding, along with many others, such as stroboscopic movement and induced movement, call for an explanation of movement perception in terms of more central events.

\section{REFERENCES}

BRINDLEY, G. S., \& MERTON, P. A. The absence of position sense in the human eye. Journal of Physiology, 1960, 153, 127-130.

HELMHOLTZ, H. Von. Physiological optics. Vol. III. New York: Dover, 1962.

POSIN, R. L. Perceptual adaptation to contingent visual-field movement: An experimental investigation of position constancy. Doctoral dissertation, Yeshiva University, 1966.

ROCK, I. The nature of perceptual adaptation. New York: Basic Books, 1966.

STOPER, A. E. Vision during pursuit movement: The role of oculomotor information. Doctoral dissertation, Brandeis University, 1967.

WALLACH, H., \& KRAVITZ, J. The measurement of the constancy of visual direction and of its adaptation. Psychonomic Science, 1965, 2, 217-218.

WALLACH, H., \& LEWIS, C. The effect of abnormal displacement of the retinal image during eye movements. Perception \& Psychophysics, 1966 1, 25-29.
YARBUS, A. L. Eye movements and vision. New York: Plenum Press, 1967.

\section{NOTES}

1. We dedicate this paper to Irvin Rock as an expression of gratitude for his valuable and very generously given advice and suggestions during the course of this work. We also want to thank L. Kaufman, A. Stoper, and L. Ganz for critically reading the manuscript; J. Hochberg and A. Mordkoff for providing necessary apparatus; and R. Sibley for help in constructing and maintaining apparatus.

2. Address: Graduate Faculty, New School for Social Research, New York, New York 100

3. It should be clear that, in the above discussion, statements about the behavior of the afterimage are restricted to only those instances where the image is viewed in a homogeneous field since, when it is viewed against a background in which there are any other visible objects, the perceived movement can always be attributed to the relative displacement of the afterimage with respect to the other objects in the field. This object-relative displacement is in itself a sufficient condition for the perception of movement.

4. Possibly the Filhene Illusion and Stoper's results represent experiences not of genuine movement but rather of a sense of object displacing in the momentary visual field (pseudo-movement). We are aware, for example, that an object while still appearing straight ahead is in the left part of the visual field when our eyes are turned far to the right.

5. Concerning the latter, if a fixated object is veridically seen to move during pursuit movements, say $10 \mathrm{deg}$ to the right, then should it not be the case that a stationary object which ends up $10 \mathrm{deg}$ to the left of the moving object should be seen as stationary?

(Accepted for publication March 27, 1969.) 